Meta

Journal des traducteurs

Translators' Journal

\title{
Phonétisation automatique du français et aménagement phonético graphique des emprunts à l'anglais
}

\section{Pierre Trescases}

Volume 32, numéro 3, septembre 1987

La fertilisation terminologique dans les langues romanes

URI : https://id.erudit.org/iderudit/004508ar

DOI : https://doi.org/10.7202/004508ar

Aller au sommaire du numéro

Éditeur(s)

Les Presses de l'Université de Montréal

ISSN

0026-0452 (imprimé)

1492-1421 (numérique)

Découvrir la revue

Citer cet article

Trescases, P. (1987). Phonétisation automatique du français et aménagement phonético graphique des emprunts à l'anglais. Meta, 32(3), 230-239.

https://doi.org/10.7202/004508ar d'utilisation que vous pouvez consulter en ligne. 


\section{PHONÉTISATION AUTOMATIQUE DU FRANCAIS ET AMÉNAGEMENT PHONETTICO-GRAPHIQUE DES EMPRUNTS À L'ANGLAIS}

PiERRE TREsCASES

Université du Nouveau-Brunswick, Fredericton, Canado

Le travail que je vais vous présenter dérive d'un projet en voie d'achèvement dont l'objectif principal était d'établir des règles graphèmes-phonèmes aussi fines que possible tenant compte de tout mot du lexique général tel qu'un dictionnaire de quelque 50000 termes comme le Petit Robert $(P R)$ peut le représenter. De telles règles permettent donc de prédire la prononciation que devrait avoir dans le système toute graphie utilisée en français.

Dans notre analyse des problèmes que peut poser la graphie des emprunts à l'anglais, eu égard à leur prononciation dans le système du français commun, ainsi que dans les propositions qui suivront concernant l'aménagement de ceux-ci sur le plan graphique et phonique, nous ne prétendons pas à l'originalité. De nombreuses et constantes propositions ont été avancées au cours des vingt dernières années dont les plus systématiques sont sans nul doute à nos yeux celles de $\mathrm{N}$. Catach (pour nous une référence aussi nécessaire que suffisante) en particulier dans son ouvrage de 1971 : Orthographe et lexicographie. Mais le faible impact de ses propositions nous incite à revenir à la charge dans le but : 1) d'éclaircir la situation, c'est-à-dire de cerner les problèmes alors même que la situation est aussi disparate que complexe ; 2) partant, d'établir des priorités dans l'aménagement phonético-graphique des emprunts non sans rapport avec la recherche d'équivalents. Ceci irait dans le sens, par exemple d'un des " critères de rejet " de l'Office de la langue française au Québec (décision $n^{\circ} 878,1980$ ) à savoir (je cite) : "Les emprunts dont l'intégration orthographique ou phonétique est difficile». (II faudrait donc pouvoir jauger cette difficulté ; 2 des 4 exemples cités (manager, management) n'étant pas très convaincants.) Dans notre présentation, nous aborderons dans l'ordre :

1. L'analyse quantitative du corpus et des données de base.

2. L'analyse qualitative des autres problèmes ainsi dégagés suivie de propositions en vue d'un aménagement linguistique.

\section{ANALYSE QUANTITATIVE}

\subsection{Corpus et données de base}

Pour des besoins de contrôle (et non de référence), nous avons utilisé le Dictionnaire de la prononciation... d'A. Martinet et $\mathrm{H}$. Walter (1973) (MW) basé lui-même sur le $P R$. C'est notre corpus de base. Un programme de phonétisation automatique révèle immédiatement les prononciations enregistrées (nous ne tiendrons compte que de la première) non conformes à la prononciation virtuelle donnée par les règles.

Ceci permet d'obtenir les données suivantes (Document $n^{\circ} l$ ) qui amènent les commentaires suivants : 
Il s'agit d'un nombre maximum qui partirait du fait a) que ing n'est pas intégré ; b) que les règles générales pour er et et produisent des prononciations "fautives " en /e/ ; que $w$ et $s h$ sont des graphèmes étrangers tout comme $n g$ et donc hors système. Cela relève d'une attitude maximaliste face au problème.

\section{AUTRES PROBLÈMES}

L'objectif principal d'un deuxième traitement des données était d'établir un répertoire des principaux problèmes (au lieu d'un recensement tous azimuts) qui puisse servir de base à une liste minimale de propositions en vue de normaliser l'adaptation phonético-graphique des emprunts à l'anglais (compte tenu du fait, encore une fois, que des propositions antérieures, parfois plus exhaustives, telles celles de N. Catach (1971), n'ont guère été prises en compte).

Il fallait donc : a) localiser les foyers de difficulté créés par les graphèmes anglais ; b) sérier statistiquement ces problèmes en fonction du nombre d'erreurs causées ; $c$ ) tenter d'évaluer le degré de difficulté (d'aucuns diraient de " perturbation ") ainsi posé dans chaque cas.

L'approche a été quelque peu différente en ce sens que nous n'avons relevé que les "fautes " manifestées dans la première prononciation enregistrée par MW par rapport à la prononciation virtuelle de nos règles de phonétisation. C'est-à-dire que nous n'avons pas retenu les formes francisées attestées par le dictionnaire et dénotant une intégration au système.

\subsection{Classification des principaux problèmes}

L'analyse statistique donne le tableau ci-après (Document $n^{\circ} 2$ ).

Un premier examen des problèmes posés par les différents graphèmes et graphies de l'anglais permet de les classer de la manière suivante (Document $n^{\circ} 3$ ).

\subsection{Impact sur les graphèmes du français et adaptation phonético-graphique}

La classification ainsi obtenue permet de dégager l'impact des graphèmes de l'anglais sur le système du français (colonnes 2 et 4 ) et d'avancer des propositions quant à leur aménagement phonético-graphique (colonne 5) (Document $n^{\circ} 4$ ).

En conclusion, 1) nous sommes conscient de ne pas avoir abordé une foule d'autres problèmes concernant l'adaptation phonétique des anglicismes ; 2) il s'agit cependant, statistiquement parlant, plus que " la pointe de l'iceberg "; 3) l'expérience devrait nous dire que " qui veut / propose le moins, peut (sans doute) le mieux "; 4) nos propositions visent essentiellement le lexique général, les 500 mots non intégrés sur le plan de la graphie ou de la phonie, du $P R$, et tous ceux qui ont eu ou auront droit de cité dans les dictionnaires d'usage dans les années 70 et 80 . Elles visent surtout les lexicographes qui devrait favoriser les adaptations ou, à tout le moins, les mettre sur le même plan que les graphies et prononciations sur le modèle d'origine et en tout cas ne plus les mentionner comme "fautives", "populaires » ou "vulgaires" alors même qu'elles sont entérinées par l'usage ; 5) nous ne considérons pas l'adaptation comme une panacée mais un complément nécessaire et parallèle à la recherche d'équivalents. Un des mérites de ces propositions concernant l'adaptation phonético-graphique des emprunts à l'anglais est peut-être précisément d'aider à orienter la recherche d'équivalents. C'est du moins ce que nous espérons. Du reste, pour citer encore une fois N. Catach (1971:118): «... l'idéal, en la matière, n'existe pas..." 


\section{DOCUMENT No 1}

Analyse des données brutes

A. Corpus de base

1. Dictionnaire de la prononciation française (basé sur le $P R$ )

50000

2. Nombre des anglicismes (selon R. Retman)

3. Nombre des anglicismes "morphologiquement visibles" (selon notre relevé)

4. Nombre maximum d'anglicismes non conforme aux règles (dont la $1^{\text {re }}$ prononciation enregistrée par MW n'est pas conforme aux règles de phonétisation)

5. Nombre réel d'anglicismes non conformes au système (non compris mots en -ing, w, sh, et, er/ r)

6. Proportion d'anglicismes francisés dans leur prononciation

$60 \%$

B. Règles de conversion graphème-morphème

1. Nombre total des règles

(y compris pour mots étrangers non anglais)

2. Nombre des règles pour anglicismes

(estimation des règles nécessaires pour les transcrire)

3. Proportion des règles pour anglicismes autres mots

C. Règles mots (" exceptions »)

1. Nombre total (anglicismes non compris)

2. Nombre nécessaire pour les anglicismes

3. Proportion des "exceptions » du système

DOCUMENT No 2

Non conformité entre la (1re) prononciation enregistrée et la prononciation virtuelle selon le système

MOTS AVEC «FAU
98
61
55
40
40
34
29
23
23
23
21
19

GRAPHIES
er
ing
$u$
a
w consonne
sh
oo
i/y
ea
in
(g)g(h)
ee




$\begin{array}{ll}15 & (\mathrm{~m}) \text { an } \\ 14 & \text { ow(n) } \\ 13 & \text { ou(n) } \\ 12 & \text { en } \\ 12 & \mathrm{j} \\ 10 & \text { oa } \\ 10 & \text { un }\end{array}$

\section{DOCUMENT NO 3}

Classification des problèmes causés par les graphies de l'anglais

1. Adoption de graphèmes et de phonèmes $n g / \eta /$ (bowling)

2. Additions de graphèmes anglais non existant dans le système correspondant à des phonèmes existant et devenant sous-graphèmes du français.

(1) $s h / s / \mathrm{CH}$

(2) $w \quad / w /$ OI

(sherry)

(sweepstake)

3. Additions de graphèmes anglais non existant dans le système et correspondant à des phonèmes anglais adaptés en français.
(1) $00 / \mathrm{u} /$
(2) $e a / i / / /$
(football)
(3) $e e / \mathrm{i} /$
(strip-tease, break)
(4) ow $/ \mathrm{o} /$
(exception /e/) (jeep)
(5) $o a / o /$
(show-boat)
(toast)

4. Modifications de la prononciation de graphèmes existant en français sous l'effet de l'anglais.
4.1 non-nasalisation
- in (pidgin)
- an (jerrycan, pullman)
- en (week-end) (on, un)
4.2 voyelles
- e(r) (sweater)
- u (club, bullfinch)
- a (bacon, hall)
- i (flirt) (dry)

4.3 consonnes

-g (gin, buggy, girl)

$-\mathrm{j}$ (jet)

4.4 diphtongue

- ou /aw/ (oun /awn/)

(out)

5. Cas du (e)

(scenic railway) 
DOCUMENT NO 4

Tableau récapitulatif de l'impact des anglicismes sur le système et des solutions proposées

\begin{tabular}{|c|c|c|c|c|}
\hline $\begin{array}{l}\text { Graphème- } \\
\text { graphie } \\
-1-\end{array}$ & $\begin{array}{l}\text { Modifications } \\
\text { apportées } \\
\text { au système } \\
-2-\end{array}$ & $\begin{array}{l}\text { Prononciation } \\
\text { en français } \\
-3-\end{array}$ & $\begin{array}{l}\text { Situation dans } \\
\text { le système } \\
\text { du français } \\
-4-\end{array}$ & Propositions \\
\hline $\mathrm{ng}$ & $\begin{array}{l}\text { addition de } \\
\text { graphème et } \\
\text { de phonème }\end{array}$ & $\begin{array}{l}\text { introduction } \\
\text { de phonème } \\
/ \mathfrak{y} /\end{array}$ & & $\begin{array}{l}\text { Phases de } \\
\text { l'aménagement : } \\
\text { 1) maintien du gra- } \\
\text { phème dans les inté- } \\
\text { grés non remplaça- } \\
\text { bles (holding), les } \\
\text { nouveaux mots tech- } \\
\text { niques et les anglicis- } \\
\text { mes culturels } \\
\text { 2) recherche d'équi- } \\
\text { valents pour les mots } \\
\text { courants ex. : bowling } \\
\text { - quilles } \\
\text { 3) recherche d'équi- } \\
\text { valents pour les ter- } \\
\text { mes techniques }\end{array}$ \\
\hline sh & $\begin{array}{l}\text { addition de } \\
\text { graphème }\end{array}$ & $/ S /$ & $\begin{array}{l}\text { sous- } \\
\text { graphème } \\
\text { de CH }\end{array}$ & $\begin{array}{l}\text { maintien de la } \\
\text { graphie (et de sa } \\
\text { prononciation } \\
\text { uniforme) }\end{array}$ \\
\hline $\begin{array}{l}\text { w } \\
\text { consonne }\end{array}$ & $\begin{array}{l}\text { addition de } \\
\text { graphème }\end{array}$ & $/ w /$ & $\begin{array}{l}\text { sous- } \\
w \text { graphème } \\
\text { de V } \\
\text { sous- } \\
\text { graphème } \\
\text { de } 01\end{array}$ & $\begin{array}{l}\text { 1) francisation des } \\
\text { mots les mieux } \\
\text { intégrés : *vagon } \\
\text { 2) maintien de } W \\
\text { ailleurs }\end{array}$ \\
\hline ou & $\begin{array}{l}\text { addition de } \\
\text { phonème }\end{array}$ & $\begin{array}{l}\text { /u/ } \\
\text { /aw/ }\end{array}$ & $\begin{array}{l}\text { - intégration } \\
\text { - correspon- } \\
\text { dance de } \\
\text { ou avec un } \\
\text { phonème } \\
\text { non indigène }\end{array}$ & $\begin{array}{l}\text { 1) Francisation de } \\
\text { la prononciation } \\
\text { 2) Recherche } \\
\text { d'équivalents } \\
\text { a) mots intégrés } \\
\text { prononcés /aw/: } \\
\text { ex. out - sortie(e) } \\
\text { b) graphies oun } \\
\text { (hors système) }\end{array}$ \\
\hline
\end{tabular}


oo

addition de $/ \mathrm{u} /$

graphème

$\mathrm{O}+\mathrm{O}$

Francisation de (système)

1) l'orthographe football

ou

* foutbal(le)

scooter — * scouteur

(Catach)

boom - boum

2) Remplacement par équivalence de tout anglicisme en oo non culturel :

looping _ boucle

ea

addition de

graphème

$e a^{/ \mathrm{i} /}$

addition de graphème $e e \rightarrow / i /$

$e^{\mathrm{I}}$

1) Francisation de ea /i/ en $i$ : strip-tease *strip-tise

2) francisation de ea / / en $e$ :

cold-cream * coldcrème

(Catach)

3) recherche d'équivalence privilégiée chandail, tricot * swêteur ; crème froide * coldcrème

\begin{tabular}{|c|c|c|c|}
\hline ee & $\begin{array}{l}\text { addition de } \\
\text { graphème }\end{array}$ & $e e \rightarrow / i /$ & $e e \rightarrow \mathbf{I}$ \\
\hline & $\begin{array}{l}\text { addition de } \\
\text { graphème } \\
\text { représentant } \\
\text { une diphtongue } \\
\text { de l'anglais }\end{array}$ & $\begin{array}{l}o w \rightarrow / o / \\
o w(n) \rightarrow / a w /\end{array}$ & $o w \rightarrow \mathrm{O}$ \\
\hline
\end{tabular}


crown-glass -

*cron-glass

Exception : clown -

*cloun (Catach)

$\begin{array}{ll}\text { addition de } \quad o a \rightarrow / 0 / \quad o a \rightarrow O \quad & \text { 1) Transcription de } \\ \text { graphème } & \text { oa par } o: \\ & \text { roast-beef }- \text { rosbif } \\ & \text { toast }-{ }^{*} \text { tost } \\ \text { (Catach) }\end{array}$

Exception : jéroboam

2) Remplacement systématique :

toast - rôtie

\begin{tabular}{|c|c|c|c|c|}
\hline in & $\begin{array}{l}\text { non } \\
\text { nasalisation }\end{array}$ & in $\rightarrow /$ in $/$ & $\begin{array}{l}\mathrm{IN} \\
\text { (système) } \\
\text { in } \mathrm{I}+\mathrm{N}\end{array}$ & $\begin{array}{l}\text { 1) Nasalisation } \\
\text { ailleurs qu'à la } \\
\text { finale : } \\
\text { badminton } \\
\text { /badmêt o/ } \\
\text { 2) Prononciation à } \\
\text { l'anglaise à la } \\
\text { finale : gin /3in/ } \\
\text { Recherche } \\
\text { d'équivalents en } \\
\text { priorité dans les } \\
\text { cas où in apparaît } \\
\text { à la finale }\end{array}$ \\
\hline an & $\begin{array}{l}\text { non } \\
\text { nasalisation }\end{array}$ & $a n \rightarrow /$ an $/$ & $a n \rightarrow \begin{array}{l}\text { AN } \\
\text { (système) }\end{array}$ & $\begin{array}{l}\text { 1) Nasalisation de } \\
\text { an dans tous } \\
\text { les mots } \\
\text { étrangers } \\
\text { (suppression du } e \\
\text { final de } \\
\text { jerricane) } \\
\text { Exception : suffixe } \\
\text { man } \\
\text { 2) Remplacement } \\
\text { systématique de } \\
\text { tous les mots en } \\
\text {-man } \\
\text { non culturels }\end{array}$ \\
\hline
\end{tabular}




\begin{tabular}{|c|c|c|c|c|c|}
\hline en & $\begin{array}{l}\text { non } \\
\text { nasalisation }\end{array}$ & $e n \rightarrow / \varepsilon n /$ & & $\begin{aligned} & \mathbf{A N} \\
& \text { (système) } \\
& \rightarrow \mathbf{E}+\mathbf{N}\end{aligned}$ & $\begin{array}{l}\text { 1) Nasalisation sauf } \\
\text { en syllabe finale } \\
\text { gentry / zãtri/ } \\
\text { 2) Non nasalisation } \\
\text { en syllabe finale } \\
\text { * wik-end /wiknd/ } \\
\text { 3) Remplacement } \\
\text { dans ces cas-là } \\
\text { *week-end - fin } \\
\text { de semaine (malgré } \\
\text { contraintes } \\
\text { syntaxiques en } \\
\text { français commun) }\end{array}$ \\
\hline on & $\begin{array}{l}\text { non } \\
\text { nasalisation }\end{array}$ & on $\rightarrow /$ on & & $\begin{array}{l}\mathrm{ON} \\
\text { (système) } \\
\rightarrow\end{array}$ & $\begin{array}{l}\text { 1) Nasalisation pour } \\
\text { tout nom commun } \\
\text { 2) Double pronon- } \\
\text { ciation } \\
\text { possible dans les } \\
\text { autres cas : } \\
\text { Exception : Boston... }\end{array}$ \\
\hline un & $\begin{array}{l}\text { non } \\
\text { nasalisation }\end{array}$ & $\begin{array}{c}\text { un /œn/ } \\
\text { un }\end{array}$ & $u n$ & $\begin{array}{l}\text { UN } \\
\text { (système) } \\
\text { EU+N } \\
\mathbf{U}+\mathbf{N}\end{array}$ & $\begin{array}{l}\text { Nasalisation } \\
\text { punch /pœe/ }\end{array}$ \\
\hline
\end{tabular}

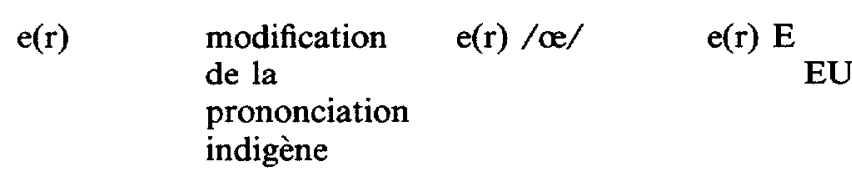

1) Alignement sur une prononciation du système de la graphie -er Ex. tanker $/ \mathrm{r} /$ (sans variation) y compris donc les anglicismes culturels :

highlander /r/

2) Recherche d'équivalent morphémique ou lexémique dans les autres cas container conteneur

N.B. : Cela équivaut à conserver $e r$ et 


\begin{tabular}{|c|c|c|c|c|}
\hline & & & & $\begin{array}{l}\text { sa prononciation } \\
\text { indigène dans } \\
\text { a) les intégrés en } \\
/ \varepsilon r /, \text { b) les } \\
\text { nouveaux emprunts, } \\
\text { c) les non } \\
\text { intégrables par } \\
\text { traduction, etc. } \\
\text { (highlander) }\end{array}$ \\
\hline $\mathbf{u}$ & $\begin{array}{l}\text { modification } \\
\text { de la } \\
\text { prononciation } \\
\text { indigène }\end{array}$ & 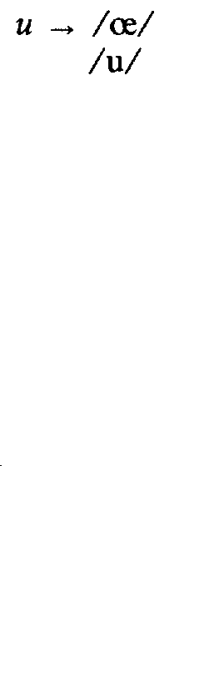 & $\begin{array}{ll}u & \mathrm{U} \\
& \text { (système) } \\
& \mathrm{EU} \\
& \mathrm{OU}\end{array}$ & $\begin{array}{l}\text { 1) Alignement de la } \\
\text { prononciation du } \\
\text { graphème sur le } \\
\text { système (pas de } \\
\text { francisation de la } \\
\text { graphie) } \\
\text { ex. club / klyb/ } \\
\text { bulldozer et non } \\
\text { bouldozeur (PR) } \\
\text { 2) Indication de } \\
\text { double prononcia- } \\
\text { tion } \\
\text { possible en syllabe } \\
\text { finale } \\
\text { surf /syrf/, /soerf/ } \\
\text { (dans l'ordre inverse } \\
\text { proposé par N. } \\
\text { Catach) }\end{array}$ \\
\hline $\mathbf{a}$ & $\begin{array}{l}\text { modification } \\
\text { de la } \\
\text { prononciation } \\
\text { indigène }\end{array}$ & $\begin{aligned} a \rightarrow & / \mathrm{e} /, / \varepsilon / \\
& / \mathrm{o} /\end{aligned}$ & $\begin{aligned} a \rightarrow & \begin{array}{l}\mathrm{A} \\
\text { (système) }\end{array} \\
& \mathrm{E} \\
& \mathrm{O}\end{aligned}$ & $\begin{array}{l}\text { 1) Alignement sur } \\
\text { les règles de } \\
\text { phonétisation : } \\
\text { ex. médecine-ball(e) } \\
\text { /a/; hall(e) /a/ } \\
\text { Exceptions : } \\
\text { a) double indication } \\
\text { de prononciation } \\
\text { pour les anglicismes } \\
\text { culturels. Ex. } \\
\text { self-made-man, } \\
\text { negro-spiritual } \\
\text { b) adaptation } \\
\text { graphique pour : } \\
\text { music-hall- } \\
\text { *music-holl } \\
\text { (musicol, Catach) } \\
\text { crawl - }{ }^{*} \text { craul } \\
\text { (Catach) } \\
\text { yacht - *yoht }\end{array}$ \\
\hline
\end{tabular}




$\begin{array}{ll}\text { modification } & i / \propto \\ \text { de la } & / \mathrm{aj} / \\ \text { prononciation } & \\ \text { indigène } & \end{array}$
$i \rightarrow \mathrm{I}$ (système) EU A + IL

1) Alignement sur les règles de phonétisation : indigène iceberg /i/

2) Prononciation à l'anglaise pour les anglicismes culturels :

ex. ragtime /aj/ 3) Remplacement systématique des anglicismes où $i$ est prononcé à l'anglaise en finale :

ex. : copyright

Exception : girl

\begin{tabular}{|c|c|c|c|}
\hline $\mathrm{g}$ & $\begin{array}{l}\text { modification } \\
\text { de la } \\
\text { prononciation } \\
\text { indigène }\end{array}$ & $\begin{array}{l}g / d z / c . / 3 / \\
(g)+e, i, y \rightarrow \\
/ g / c . / 3 / \\
(\mathrm{gh}) \\
(\mathrm{gg})\end{array}$ & $\begin{array}{c}g \rightarrow G \text { au } \\
\text { au lieu de } \mathbf{J} \\
\mathrm{D}+\mathbf{J}\end{array}$ \\
\hline
\end{tabular}

1) Alignement sur les règles de phonétisation : ex. gin/3in/ 2) Remplacement systématique des anglicismes où (g) $\mathrm{g}(\mathrm{h})$ suivi(s) de $\mathrm{e}, \mathrm{i}, \mathrm{y}$ correspondent à une occlusive en français Ex. getter, dinghy, jigger

\begin{tabular}{|c|c|c|c|c|}
\hline $\mathrm{j}$ & $\begin{array}{l}\text { modification } \\
\text { de la } \\
\text { prononciation } \\
\text { indigène }\end{array}$ & $j \rightarrow / \mathrm{d} 3 /$ & $\begin{array}{c}j \rightarrow \underset{\text { (système) }}{\mathrm{J}} \\
\qquad+\mathrm{J}\end{array}$ & $\begin{array}{l}\text { 1) Alignement sur } \\
\text { les règles de } \\
\text { phonétisation } \\
\text { Ex. jet / zहt/ }\end{array}$ \\
\hline $\mathrm{ch}^{*}$ & $\begin{array}{l}\text { modification } \\
\text { de la } \\
\text { prononciation } \\
\text { indigène }\end{array}$ & $c h \rightarrow / \mathrm{t} s /$ & $\begin{array}{c}\text { ch } \underset{\text { (système) }}{\mathrm{CH}} \\
\mathrm{T}+\mathrm{CH}\end{array}$ & $\begin{array}{l}\text { 1) Alignement sur } \\
\text { les règles de } \\
\text { phonétisation } \\
\text { Ex. : sandwich } \\
\text { /sadwis/ }\end{array}$ \\
\hline
\end{tabular}

